The Astrophysical Journal, 619: L127-L130, 2005 FEBruARY 1

Preprint typeset using $\mathrm{LT}_{\mathrm{E}} \mathrm{X}$ style emulateapj v. 6/22/04

\title{
FIRST MEASUREMENTS OF THE POLARIZATION OF THE COSMIC MICROWAVE BACKGROUND RADIATION AT SMALL ANGULAR SCALES FROM CAPMAP
}

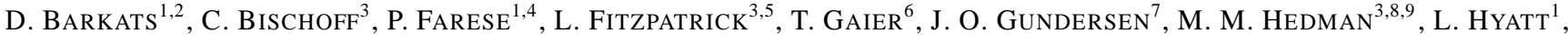

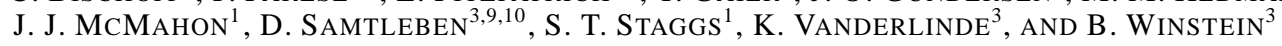

\begin{abstract}
Polarization results from the Cosmic Anisotropy Polarization MAPper (CAPMAP) experiment are reported. These are based on $433 \mathrm{hr}$, after cuts, observing a $2 \mathrm{deg}^{2}$ patch around the north celestial pole with four 90 $\mathrm{GHz}$ correlation polarimeters coupled to optics defining $4^{\prime}$ beams. The $E$-mode flat band-power anisotropy within $l=940_{-300}^{+330}$ is measured as $66_{-39}^{+53} \mu \mathrm{K}^{2}$; the $95 \%$ confidence level upper limit for $B$-mode power within $l=1050_{-520}^{+590}$ is measured as $38 \mu \mathrm{K}^{2}$.

Subject headings: cosmology: cosmic microwave background - cosmology: observations
\end{abstract}

\section{INTRODUCTION}

The cosmic microwave background (CMB) is arguably the most fruitful source of cosmological information. Its spectrum has been measured with extraordinary precision (Fixsen et al. 1997) and its spatial anisotropy has been very well characterized, with the Wilkinson Microwave Anisotropy Probe (WMAP; Bennett et al. 2003a) providing the most comprehensive results. There are two distinct patterns to the polarization of the $\mathrm{CMB}$, conventionally termed $E$ - and $B$ modes. The former have recently been detected at the degree scale (Kovac et al. 2002; Kogut et al. 2003); they arise from the same density perturbations that dominate the temperature anisotropies. The latter, which can result from the lensing of $E$-modes due to the intervening matter distribution or from primordial gravity waves, are expected to be far smaller. Characterization of the $E$-mode power spectrum is important for testing the understanding of the origin of the CMB as well as for breaking degeneracies in cosmological parameter determinations (e.g., Bucher et al. 2001; Hu 2003).

In this Letter we present results from the first season of CAPMAP (Cosmic Anisotropy Polarization MAPper), an effort to characterize the $E$-mode polarization at $90 \mathrm{GHz}$, where foregrounds are expected to be small, and at $4^{\prime}$, where the polarization is expected to be near its maximum. In $\S \S$ 2 and 3 we describe the instrument, its characterization and calibration, and the observations. In subsequent sections we summarize the reduction of the data $(\S[4)$, the analysis $(\S 5$, and the systematic studies $(\S 6$. We conclude this Letter with a discussion of the results and their implications ( $\$ 7$.

1 Department of Physics, Princeton University, Joseph Henry Laboratories, Jadwin Hall, P.O. Box 708, Princeton, NJ 08544-0708.

2 Current address: Department of Physics, California Institute of Technology, 1200 East California Boulevard, MS 59-33, Pasadena, CA 91125.

3 Kavli Institute for Cosmological Physics and Enrico Fermi Institute, University of Chicago, 5641 South Ingleside Avenue, Chicago, IL, 60637.

${ }^{4}$ Dicke Fellow.

5 Current address: Department of Physics, Harvard University, 17A Oxford Street, Cambridge, MA 02138.

${ }^{6}$ Jet Propulsion Laboratory, California Institute of Technology, 4800 Oak Grove Drive, Pasadena, CA 91109.

7 Department of Physics, University of Miami, James L. Knight Physics Building, 1320 Campo Sano Drive, Coral Gables, FL 33146.

${ }^{8}$ Current address: Department of Astronomy, Cornell University, Space Sciences Building, Ithaca, NY 14853-6801.

${ }^{9}$ Kavli Fellow.

${ }^{10}$ Corresponding author: dorothea@kicp.uchicago.edu.

\section{INSTRUMENT}

The CAPMAP instrument is described in detail in Barkats et al. (2005); here its most important features are given. In the winter of 2003, four W-band (84-100 $\mathrm{GHz}$ ) polarimeters (A-D) were installed in the focal plane of the $7 \mathrm{~m}$ Cassegrain antenna (Chu et al. 1978) at the Lucent Technologies facility in Crawford Hill, New Jersey (W74 $11^{\prime} 11^{\prime \prime}, \mathrm{N} 40^{\circ} 23^{\prime} 31^{\prime \prime}$ ). The horn and meniscus lens system attached to each polarimeter underilluminated the telescope mirrors with an edge taper of $-35 \mathrm{~dB}$ on the primary, resulting in a final beam of $3^{\prime} .88$ FWHM $\left(\sigma_{B}=1^{\prime} .65\right)$ with an ellipticity less than $3 \%$. The polarimeters were housed inside a single cryostat, nominally arranged so that A (C) observed $0^{\circ} .25$ below (above) and B (D) $0^{\circ} .25$ left (right) of the optical axis of the telescope.

Heterodyne analog correlation polarimeters that multiply two orthogonal components of the incident electric field produced an output proportional to one linear combination of the Stokes parameters $Q$ and $U$. The two components derive from an ortho-mode transducer (OMT) oriented with one axis horizontal. The polarimeter output is thus proportional to the difference in power for field components aligned $\pm 45^{\circ}$ to the horizontal in the focal plane. The first-stage low noise amplifiers (LNAs) in each arm are InP HEMT monolithic microwave-integrated circuits developed by JPL and Northrop Grumman Space Technology (Weinreb et al. 1999). A mechanical refrigerator cools the horns, the OMT, and the LNAs to $\leq 40 \mathrm{~K}$. One local oscillator inside the cryostat supplies the polarimeters with the $82 \mathrm{GHz}$ to downconvert the 84-100 GHz signals to an intermediate frequency (IF) of $2-18 \mathrm{GHz}$. The oscillator signal in one arm of each polarimeter is phase-switched at $4 \mathrm{kHz}$, well above the $1 / f$ knee of the amplifiers. A detector diode monitors the total power in each arm. The phase-switched IF radiation is split into three $4 \mathrm{GHz}$-wide subbands (S0, S1, S2) prior to multiplication, yielding then 12 polarization and eight total power channels.

The polarimeter outputs are digitized (24 bits, $100 \mathrm{kHz}$ ) with a $\Sigma \Delta$ analog-to-digital converter (ICS-610); digitally demodulated data are recorded at $100 \mathrm{~Hz}$. The $100 \mathrm{kHz}$ samples, averaged over 40 phase switches, are recorded to produce quadrature data sets, demodulated out of phase with the phase switch, which contain no celestial signal.

\section{OBSERVATIONS AND CALIBRATION}


Between 2003 February 18 and April 6, 541 hr of CMB data were recorded. Calibration data were taken before, regularly throughout, and after the season.

For the CMB observation the telescope's optical axis moved through the north celestial pole (NCP) with an $8 \mathrm{~s}$ period by $\pm 0^{\circ} .53$ at constant elevation. Polarimeter offsets from the optical axis are given in Table 11 the sampled region is a circle of diameter $1^{\circ} .6$ centered on the NCP, with the inner circle of diameter $0^{\circ} .14$ missing. This scan strategy yields quite uniform $Q / U$ coverage over the region.

TABLE 1

POLARIMETER POSITION OFFSETS ${ }^{a}$

\begin{tabular}{lllll}
\hline & $\mathrm{A}$ & $\mathrm{B}$ & $\mathrm{C}$ & $\mathrm{D}$ \\
\hline \hline co-elevation & -0.022 & -0.247 & -0.025 & 0.200 \\
cross-elevation & -0.286 & -0.066 & 0.162 & -0.059
\end{tabular}

${ }^{a}$ The polarimeter offsets from the optical axis are given in degrees. For the CMB observations, the constant-elevation scan was centered with the optical axis at $\left(-0^{\circ} .03,-0^{\circ} .09\right)$ from the NCP.

The pointing solution, determined with Jupiter observations, was refined to $\pm 30^{\prime \prime}$ using 150 observations of astrophysical sources. The relative offsets, beam sizes and the total power calibration were determined from the observations of Jupiter in the total power channels.

Noise temperatures were estimated from several elevation scans $\left(20^{\circ}-90^{\circ}\right)$. They ranged between 60 and $70 \mathrm{~K}$ and agreed with laboratory cold load tests.

For the determination of polarized gains, a nutating plate was installed in front of the secondary mirror to produce a modulated polarized signal; this gave calibrations with an overall uncertainty of $10 \%$ and a $3 \%$ relative uncertainty among the channels. Frequent scans of the polarized source Tau A near parallactic angle $110^{\circ}$ (where the signal in the receivers is maximal) provided a good calibration check (Barkats et al. 2005). Polarimeter sensitivities are summarized in Table 2

TABLE 2

POLARIMETER CHARACTERISTICS

\begin{tabular}{|c|c|c|c|c|c|}
\hline \multirow[b]{2}{*}{ Channel } & \multicolumn{4}{|c|}{ Polarimeter $^{a}$} & $\overline{\nu_{e f f}^{b}}$ \\
\hline & $\mathrm{A}$ & $\mathrm{B}$ & $\mathrm{C}$ & $\mathrm{D}$ & \\
\hline So & 3.2 & 3.4 & 2.9 & 3.1 & 87 \\
\hline S1 & 2.1 & 2.1 & 2.1 & 2.2 & 92 \\
\hline $\mathrm{S} 2$ & 2.7 & 2.0 & 3.5 & 2.5 & 96 \\
\hline
\end{tabular}

${ }^{a}$ Sensitivities in $\mathrm{mK} \sqrt{s}$ in thermodynamic units

${ }^{b}$ Typical central frequency in $\mathrm{GHz}$

The calibration was corrected for slow few kelvin variations in the IF electronics and for a varying phase between the digitization and phase switch clocks. The IF gains changed by up to $3 \% \mathrm{~K}^{-1}$ but the temperature was stable to $1 \mathrm{~K}$ for $70 \%$ of the data. The clock correction ranged from 0.9 to 1 and varied by at most $0.003 \mathrm{hr}^{-1}$. The calibration for two of the channels (DS0, DS2) was adjusted by $\sim 10 \%$ to compensate for gain compression.

\section{DATA REDUCTION}

The CMB data were divided into $40 \mathrm{~s}$ periods; data within each were divided into $20 \sim 3^{\prime}$-wide, nearly uniformly

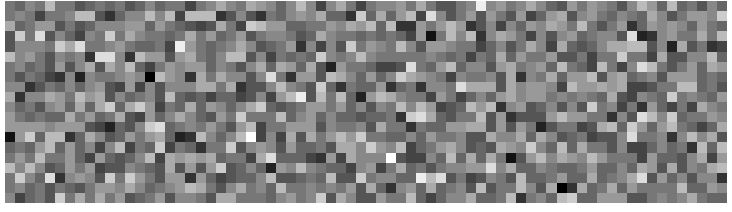

FIG. 1. - The $20 \times 72$ data vector for receiver C. The scale ranges between $\pm 210 \mu \mathrm{K}$; the average unchopped error for each element is $64 \mu \mathrm{K}$. These pixels are spaced approximately 0.8 beamwidths in azimuth; in the LST direction, their spacing ranges from about 0.1 to 1 beam width depending on the azimuthal position.

populated azimuthal bins, discarding the $9 \%$ of the data at the turnarounds. Means and standard errors calculated in each bin were carried forward in the analysis. Varying the period between 16 and $40 \mathrm{~s}$ did not alter the final results.

A second-order polynomial was fitted to the azimuthal samples in each $40 \mathrm{~s}$ period and removed. The polarimetry channels reject the common mode intensity by more than $23 \mathrm{~dB}$, but their residual sensitivity to the atmospheric temperature, which varied daily by $10 \mathrm{~K}$, dominated the intercept terms. The linear terms were evident when co-added over an hour, revealing slopes as large as $500 \mu \mathrm{K} \mathrm{deg}^{-1}$, with variations up to $200 \mu \mathrm{K} \mathrm{deg}^{-1} \mathrm{day}^{-1}$. The quadratic terms were even smaller and more stable.

Abnormally high fluctuations $(>4 \sigma)$ in the polarized channels or unusual changes in the ratios between different total power channels were observed for $20 \mathrm{hr}$ of data. Both symptoms result from ice formation on the cryostat windows so these data were removed. The five most sensitive total power channels were used to identify bad weather with a cut on their fractional deviations. Only periods that passed the cut for all five channels were retained, eliminating $88 \mathrm{hr}$ and leaving $433 \mathrm{hr}$ for the final data sample. Ten percent variations in this cut level changed the result within statistics.

Collapsing all polarization data into the 20 azimuthal bins reveals scan synchronous structures (SSSs) with rms levels from 10 to $26 \mu \mathrm{K}$; from a $90 \mathrm{GHz}$ side lobe beam map, these are likely due to ground pickup. Dependences on variables like system temperature, local sidereal time (LST), or ambient temperature were studied. Only BS1 and BS2 showed such a dependence, varying with the ambient temperature. The mean of the data in each azimuthal bin was removed, and residual effects that could arise from correlations to ambient temperature are modeled as described below. The channel with the largest structure and variation (BS2) is dropped with negligible change in the results but with a significant reduction in the systematic uncertainty.

After structure removal, corrections for atmospheric absorption (typically 20\%) derived from the total power channels, were applied. The corrected periods, already parsed into azimuth bins, were binned into 72 LST bins, yielding $72 \times 20=1440$ element data vectors for each of the 12 channels. The weighted averages of the three frequency channels (which have correlations less than 1\%) were used in the likelihood analysis. One resulting data vector is displayed in Figure 1

\section{DATA ANALYSIS}

A Bayesian likelihood analysis was performed by maximizing the likelihood,

$$
\mathcal{L}=\frac{1}{\sqrt{\operatorname{det}(C)}} \exp \left\{-\mathbf{x}^{T} C^{-1} \mathbf{x} / 2\right\}
$$


where $\mathbf{x}$ is the data vector with $4 \times 1440$ entries and $C$ is the covariance matrix. The vector $\mathbf{x}$ sums the signal $(s)$ and receiver noise $(n)$ terms: $x_{i}=A_{i j} s_{j}+n_{i}$, where $A_{i j}$ determines the linear combination of $Q\left(s_{1}\right)$ and $U\left(s_{2}\right)$ in pixel $i$. Similarly, the covariance matrix comprises signal $\left(C_{T}\right)$ added to noise $N$ : $C_{i j}=<x_{i} x_{j}>=C_{T, i j}+N_{i j}$, where $N_{i j}=n_{i}^{2} \delta_{i j}$, with typical noise $n_{i} \approx 60 \mu \mathrm{K}$. The signal covariance depends on the multipole moments for $E$ - and $B$-modes, $C_{l}^{E}$ and $C_{l}^{B}$ :

$$
C_{T, i j}=\sum_{l} \frac{2 l+1}{4 \pi}\left(C_{l}^{E} W_{l, i j}^{E}+C_{l}^{B} W_{l, i j}^{B}\right) .
$$

Here, the window function matrices $W_{l}^{X}$ account for (1) beam functions $B_{l}^{2} \approx \exp \left(-l^{2} \sigma_{B}^{2}\right),(2)$ the different pixel areas in the data vector, and (3) the conversion from $(Q, U)$ to $X=(E, B)$ (Zaldarriaga \& Seliak 1997). Figure 2 shows the trace of receiver C's $E$-mode window function, similar to those of the other receivers. The likelihood is maximized with respect to one to three variables that parameterize $C_{l}^{E}$ and/or $C_{l}^{B}$, as described below.

The signal-to-noise ratio eigenmode method was used (e.g., Bond et al. 1998): transformations into the basis where the noise matrix is white and then into the eigenbasis of the transformed $C_{T}$ are performed. Removing offsets every $40 \mathrm{~s}$ and subtracting SSSs transform $\mathbf{x}$ to $D \mathbf{x}$. Accordingly, $C$ is transformed to $D C D^{T}$, and the analysis is continued in the subspace onto which $D$ projects.

The code was exercised extensively with simulated data sets. Realizations with noise levels from that of the data to 20 times smaller were superposed on CMB realizations of the concordance model (Spergel et al. 2003). The likelihood estimator was found to be unbiased; however, at the noise level of the data, the loss of sensitivity from the offset removals is a factor of 2 .

Four likelihood evaluations were made: first by taking $C_{l}^{E}$ as a multiplier of the concordance model, with $C_{l}^{B} \equiv 0$; second by taking $l(l+1) C_{l}^{E}$ to be a constant (flat band-power), with $C_{l}^{B} \equiv 0$; third by simultaneously taking $C_{l}^{E}$ as a multiplier to the concordance model and $l(l+1) C_{l}^{B}$ as a constant; and fourth by taking $C_{l}^{E}$ to comprise three flat band-power levels, in the $l$ bins (2-500/501-1500/1501-2900), with $C_{l}^{B} \equiv 0$ (three-band analysis). Fit results are summarized in Table 3 Effective $l \mathrm{~s}$ are calculated according to Steinhardt (1995) and quoted with the central $68 \%$ region of $W_{l} / l$.

The various likelihood evaluations yield a $2 \sigma$ detection of the $E$-mode signal, with no $B$-mode signal or significant dependence on frequency observed. Results from the threeband analysis are shown in Figure 2] The upper band has negligible correlation with the other two. For the lower two bands the marginalized results from integrating over their likelihood contours are displayed. The marginalization shifted the peak of the middle band down by $6 \%$, increasing its width by $12 \%$. A fit of the middle $l$-band's likelihood to an offset lognormal distribution (Bond et al. 2000) gives $66 \mu \mathrm{K}^{2}$ with a variance of $\left(44 \mu \mathrm{K}^{2}\right)^{2}$ and a noise related offset of $93 \mu \mathrm{K}^{2}$.

\section{EVALUATION OF SYSTEMATIC UNCERTAINTIES}

The three most important sources of systematic uncertainty were associated with the telescope pointing, the scan synchronous residual structure and the relative gains. Their impact was evaluated on fits of $E$-mode power to a multiplier of the concordance model; selected studies confirmed that
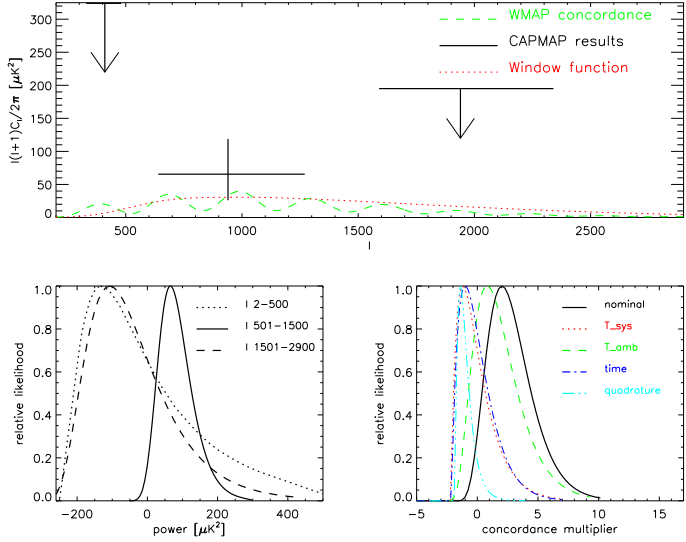

FIG. 2.- Likelihood results. Top: Results from the three-band analysis, the concordance model (long dashed line), and the trace of the window function $W_{l}^{E}$, as defined in equation 2, for receiver $\mathrm{C}$, in arbitrary units (dotted line). For the outer bands, 95\% confidence level upper limits are displayed. Lower left: Individual marginalized likelihood curves for the three bands. Lower right: Concordance model fitted to the data and several null maps as described in the text.

uncertainties estimated with those fits apply to the three other fitting methods.

The effect of possible mispointing was determined by varying the global pointing solution within its uncertainty, $\sim 10 \%$ of a beamwidth. The $1 \sigma$ shifts in azimuth and elevation were added in quadrature, resulting in an uncertainty of $12 \%$.

Effects of the SSSs were simulated. It was determined separately in each channel for two halves of the data, divided according to ambient temperature and its extracted (linear) variation. Multiple simulations were made with structure added to the CMB according to the ambient temperature in each period. This shifted the result upward by on average 0.1 (times concordance), and the spread, as the underlying CMB was varied, was \pm 0.1 . The uncertainty is then conservatively taken to be \pm 0.2 (corresponding to a $10 \%$ uncertainty).

The effect of relative gain uncertainties was estimated from the scatter in the result with the gain of each of the 11 polarimeters chosen randomly within the estimated $\pm 3 \%$ uncertainty, leading to an uncertainty of $6 \%$.

Three other sources of uncertainty were studied. The beam size was uncertain to $2 \%$; varying it by even $10 \%$ leads to less than a $3 \%$ change in the result. As mentioned earlier, the polarimeters have a weak response to temperature anisotropies $(-23 \mathrm{~dB})$, and the optics produces a small quadrupole response at scales smaller than the beam $(-12 \mathrm{~dB})$. Simulations show that these also lead to negligible effects (see also Hu et al. 2003).

Several checks of the robustness of the result were performed. Excluding single radiometers or $25 \%$ data samples gave consistent results. Difference maps were created by splitting the data into two parts according to high and low system and ambient temperatures and time. Data vectors were produced for each half $\left(D_{1}\right.$ and $\left.D_{2}\right)$ and the likelihood was evaluated on $\left(D_{1}-D_{2}\right) / 2$. These likelihoods, together with that for the quadrature data, are shown in Figure 2, none show a signal. Finally, a parallel analysis was performed. Offsets were removed on a $4 \mathrm{~s}$ timescale, and an independent code implemented a different pixelization scheme and a different means of evaluating the associated 
covariance matrix and nulling out the appropriate modes. It was used to obtain a multiplier to concordance and gave a consistent result.

Adding the three main uncertainties in quadrature, the overall systematic uncertainty becomes $17 \%$. The $10 \%$ overall gain uncertainty contributes an additional $20 \%$ without reducing the significance of the detection.

TABLE 3

CAPMAP RESULTS ${ }^{a}$

\begin{tabular}{|c|c|c|c|c|c|}
\hline \multicolumn{6}{|c|}{$E$-mode multiplier to concordance, $l=1120_{-520}^{+560}, C_{l}^{B} \equiv 0$} \\
\hline frequency & all $^{a}$ & S0 & S1 & S2 & \\
\hline result $^{b}$ & $2.0_{-1.5}^{+2.1}$ & $3.9_{-4.8}^{+7.3}$ & $-0.1_{-1.8}^{+2.8}$ & $9.4_{-6.1}^{+8.8}$ & \\
\hline \multicolumn{6}{|c|}{ Fits to flat band-power, $C_{l}^{B} \equiv 0$ except for fit for $B$} \\
\hline & $\mathrm{B}^{c}$ & $\mathrm{E}$ & $\mathrm{E} 1^{d}$ & $\mathrm{E} 2^{d}$ & $\mathrm{E} 3^{d}$ \\
\hline$l$ & $1050_{-520}^{+590}$ & $1120_{-520}^{+560}$ & $410_{-80}^{+70}$ & $940_{-300}^{+330}$ & $1940_{-350}^{+400}$ \\
\hline result ${ }^{a}$ & $<38 \mu \mathrm{K}^{2}$ & $31_{-27}^{+39} \mu \mathrm{K}^{2}$ & $<324 \mu \mathrm{K}^{2}$ & $66_{-39}^{+53} \mu \mathrm{K}^{2}$ & $<195 \mu \mathrm{K}^{2}$ \\
\hline
\end{tabular}

${ }^{a}$ Simultaneous fit with $B$ also gives $2.0_{-1.5}^{+2.1}$

${ }^{b}$ With $68 \%$ interval of highest posterior density or $95 \%$ upper limit

${ }^{c}$ Fitted simultaneously with $E$

${ }^{d}$ Three-band analysis

\section{DISCUSSION}

Evidence of polarization anisotropy in the $\mathrm{CMB}$ in the multipole region around $l=1000$, where the $E$-mode power spectrum is expected to peak, has been presented. Signals of increasing significance are seen when fitting for (1) a flat band-power, (2) a multiplier of the expected concordance power spectrum, and (3) power in a band centered at these high $l$-values. The $E$-mode signal does not change when fitting simultaneously for both $E$ - and $B$-modes, and the latter are not detected. Here the possible contaminations to these results are discussed together with their significance.

Foregrounds are not expected to be significant. From Finkbeiner et al. (1999) dust maps and WMAP synchrotron maps (Bennett et al. 2003b) around the NCP, temperature anisotropies of 4.4 and $1.3 \mu \mathrm{K}$, respectively, are expected. Even with pessimistic assumptions about polarization fractions and how these foregrounds extrapolate to CAPMAP's angular scales, their contributions are well below the observed signal.

There are no deep source maps exactly at the NCP. A survey at $90 \mathrm{GHz}$ (Holdaway et al. 1994) extrapolates 4400 sources brighter than $100 \mathrm{mJy}$ over the entire sky, implying a $20 \%$ chance for one to be in the CAPMAP field. From the instrument response $\left(3 \mu \mathrm{K} \mathrm{mJy}^{-1}\right)$, such a source would contribute at the $20 \mu \mathrm{K}$ level in 1 pixel if it had the same high polarization as Tau A (7.5\%). Effects on the polarization power spectra, along with those from weaker but more copious sources, are negligible. Conclusions from a dedicated source survey near the NCP (Leitch et al. 2000) are similar.

Adding the systematic uncertainty in quadrature to the result in the middle 1-band, a null result is excluded at just over the $2 \sigma$ level. Including the overall calibration uncertainty, this result is consistent with the concordance model within $72 \%$ of the highest posterior density. This result at $90 \mathrm{GHz}$ is consistent with the very recent results from the Degree Angular Scale Interferometer and Cosmic Background Imager at $30 \mathrm{GHz}$ (Leitch et al. 2004; Readhead et al. 2004).

We thank Tom Crawford, Wayne Hu, Norm Jarosik, Stephan Meyer, Lyman Page, and David Spergel for many helpful discussions and Michelle Yeh and Eugenia Stefanescu for help in the data collection. We thank Lucent Technologies for use of the $7 \mathrm{~m}$ telescope and Bob Wilson, Greg Wright and Tod Sizer for their assistance with it. This work was supported by NSF grants PHY-9984440, PHY-0099493, PHY-0355328, AST-0206241 and PHY-0114422 and by the Kavli Foundation. Portions of this work were carried out at the Jet Propulsion Laboratory, California Institute of Technology, operating under a contract from the National Aeronautics and Space Administration.

\section{REFERENCES}

Barkats, D., et al. 2005, ApJS, submitted

Bennett, C. L., et al. 2003a, ApJS, 148, 1

Bennett, C. L., et al. 2003b, ApJS, 148, 97

Bond, J. R., Jaffe, A. H., \& Knox, L. 1998, Phys. Rev. D, 57, 2117

-. 2000, ApJ, 533, 19

Bucher, M., Moodley, K., \& Turok, N. 2001, Phys. Rev. Lett., 87, 191301

Chu, T. S., Wilson, R. W., England, R. W., Gray, D. A., \& Legg, W. E. 1978, The Bell System Technical Journal, 57, 1257

Finkbeiner, D. P., Davis, M., \& Schlegel, D. J. 1999, ApJ, 524, 867

Fixsen, D. J., Hinshaw, G., Bennett, C. L., \& Mather, J. C. 1997, ApJ, 486, 623

Holdaway, M. A., Owen, F. N., \& Rupen, M. P. 1994, MMA Memo 123, http://www.alma.nrao.edu/memos/html-memos/alma123/

Hu, W. 2003, Annals of Physics, 303, 203

Hu, W., Hedman, M. M., \& Zaldarriaga, M. 2003, Phys. Rev. D, 67, 043004

Kogut, A. , et al. 2003, ApJS, 148, 161
Kovac, J. M., Leitch, E. M., Pryke, C., Carlstrom, J. E., Halverson, N. W., \& Holzapfel, W. L. 2002, Nature, 420, 772

Leitch, E. M., Kovac, J. M., Halverson, N. W., Carlstrom, J. E., Pryke, C., \& Smith, M. W. E. 2004, ApJ, submitted iastro-ph/0409357

Leitch, E. M., Readhead, A. C. S., Pearson, T. J., Myers, S. T., Gulkis, S., \& Lawrence, C. R. 2000, ApJ, 532, 37

Readhead, A. C. S., et al. 2004, Science, 306, 836

Spergel, D. N., et al. 2003, ApJS, 148, 175

Steinhardt, P. J. 1995, in Particle and Nuclear Astrophysics and Cosmology in the Next Millenium, ed. E. W. Kolb \& R. D. Peccei (Singapore: World Scientific), 51

Weinreb, S., Lai, R., Erickson, N., Gaier, T., \& Wielgus, J. 1999, in IEEE

MTT-S, Int. Microwave Symp. Digest, 101

Zaldarriaga, M., \& Seljak, U. 1997, Phys. Rev. D, 55, 1830 